\title{
How to increase earthquake and home fire preparedness: the fix-it intervention
}

\author{
Helene Joffe $^{1} \cdot$ Gabriela Perez-Fuentes $^{1} \cdot$ Henry W. W. Potts ${ }^{2}$ • \\ Tiziana Rossetto ${ }^{3}$
}

Received: 3 January 2016/Accepted: 4 August 2016/Published online: 16 August 2016

(C) The Author(s) 2016. This article is published with open access at Springerlink.com

\begin{abstract}
Published, evaluated community intervention studies concerning natural hazard preparedness are rare. Most lack a rigorous methodology, thereby hampering the development of evidence-based interventions. This paper describes the rationale and methodology of a cross-cultural, longitudinal intervention study on earthquake and home fire preparedness, termed fix-it. The aim is to evaluate whether and how the intervention brings about behaviour change in the targeted communities in two coastal cities with high seismic risk: Seattle, USA and Izmir, Turkey. Participants are adult residents of these cities. The intervention group attends a 6-h workshop, which focuses on securing items in the household. The control group does not attend the workshop. All participants complete baseline and post-intervention, as well as 3- and 12-month follow-up assessments. The primary outcome measure is an observational measure of nine preparedness items for earthquake and fire in participants' homes. This is evaluated alongside participants' self-reports concerning their preparedness levels. Secondary outcomes are changes in levels of self-efficacy, perceived outcome, trust, corruption, empowerment, anxiety and social cohesion. Results from the first of the studies, conducted in Seattle in September 2015, indicate that while the fix-it intervention is effective, in the longer term, multi-hazard preparedness is increased by the mere act of going into people's homes to observe their preparedness levels along with assessing self-reported preparedness and sociopsychological orientation towards natural hazards. This protocol and study aim to augment the empirical literature on natural hazard preparedness, informing
\end{abstract}

Electronic supplementary material The online version of this article (doi:10.1007/s11069-016-2528-1) contains supplementary material, which is available to authorized users.

Helene Joffe

h.joffe@ucl.ac.uk

1 Department of Clinical, Educational and Health Psychology, University College London (UCL), 26 Bedford Way, London WC1H 0AP, UK

2 Institute for Health Informatics, University College London (UCL), 222 Euston Road, London NW1 2DA, UK

3 Department of Civil, Environmental and Geomatic Engineering, University College London (UCL), Gower Street, London WC1E 6BT, UK 
national and international policy on delivery of evidence-based community interventions to promote multi-hazard preparedness in households.

Keywords Community preparedness intervention · Multi-hazard preparedness · Earthquake preparedness - Fire preparedness - Behaviour change

\section{Introduction}

Globally, natural disasters have claimed an average of 68,000 lives per year over the past 20 years, with earthquakes and tsunamis killing more people than all other types of disasters together: they claimed approximately 750,000 lives between 1994 and 2013 (CRED and UNISDR 2015). Furthermore, natural disasters cost an average of US\$250-300 billion globally each year (Maskrey and Safaie 2015). Community preparedness for natural hazards is critical in reducing the risk of injury, death and damage to homes; it also facilitates coping with the temporary disruption caused by the hazard (Paton 2000). Cities and communities that are prepared for several hazards are more resilient than those that prepare for a single hazard (Scawthorn et al. 2006b). Consequently, the multi-hazard approach is gaining popularity in the field of natural hazard preparedness (Scawthorn et al. 2006a). Disaster preparedness measures can range from securing heavy objects, structural retrofitting and storing food and water, to having insurance, an emergency kit and communication and evacuation plans.

While significant efforts have been made in recent years to increase public awareness and to train communities in hazard preparedness, such work has not proven sufficient in increasing the adoption of mitigating measures. Community preparedness for natural hazards remains low across cultures (Ballantyne et al. 2000; Lindell and Whitney 2000; Paton 2000), even among those where awareness of the natural hazard risk is high (Joffe et al. 2013; Karanci et al. 2005; Lindell 2013; Solberg et al. 2010)

Despite the importance of natural disaster preparedness interventions in reducing personal, social and economic losses, the literature on such interventions is scarce and its methodology is often not well documented (Perez-Fuentes and Joffe 2015). There are a wide range of mass media and internet-based natural disaster preparedness engagement methods, yet their documentation and evaluation are rare (Verrucci et al. 2016). When preparedness campaigns and interventions are evaluated, most are vaguely described. Furthermore, those interventions that are evaluated tend to be based on self-report measures alone, where social desirability threatens the validity of the results (Eisenman et al. 2009; Glik et al. 2014; Karanci et al. 2005). Lastly, few of the existing published interventions on earthquake and home fire preparedness have been proven to demonstrate increased disaster preparedness behaviours at the household level. Therefore, more explicit and better designed natural hazard preparedness interventions are needed so that they can be replicated and improved. The long-term objective is to expose publics only to evidence-based interventions. This paper aims to describe one such intervention, which was devised on the basis of the existing psychological literature concerning successful natural hazard preparedness and health behaviour change interventions. 


\subsection{Effective intervention studies on earthquake and fire preparedness}

Despite their scarcity, there are a number of examples of successful interventions and campaigns regarding community preparedness that provide a transparent methodology. Findings from these studies inform the present intervention. Overall, disaster preparedness interventions have proven successful in affecting adjustment measures when including hands-on training (Miller et al. 2014), drills (Simpson 2002), face-to-face interactions as opposed to sending a pamphlet or internet-based information, targeting social empowerment, a process of increasing personal, interpersonal or political power so that individuals can take action to improve their life situations (Paton et al. 2003) and community cohesion (Eisenman et al. 2009; Glik et al. 2014), defined as a "set of attitudes and norms that include trust, a sense of belonging, and the willingness to participate and help, as well as their behavioural manifestations" (Chan et al. 2006).

Based on additional evidence, other factors must also be built into interventions to make them effective. Proven predictors of preparedness are: perceived self-efficacy, that is, beliefs regarding personal capacity to act effectively, which influences the number and quality of plans, effort and perseverance in risk reduction behaviours (Paton et al. 2003); collective efficacy, defined as people's shared beliefs in their collective power (Paton and Johnston 2015); perceived outcome expectancy, the perception of whether ones' actions will reduce the problem or threat (Paton et al. 2003); behavioural intention, which refers to the motivation to act and precedes adjustment adoption (Paton et al. 2000, 2010); critical awareness, defined as the frequency of talking and thinking about a specific hazard (Paton and Johnston 2015; Paton et al. 2005); sense of community, that is, feelings of belonging and attachment to people and places (Bishop et al. 2000; Paton 2000); community participation, which refers to individuals who form groups to collaborate with management agencies (Karanci and Aksit 2000; McGee and Russell 2003; Paton and Johnston 2015; Paton et al. 2005; Tierney et al. 2001); and trust in authorities, defined as trust in various sources of information (Dillon and Phillips 2001; Paton and Johnston 2015). In addition to targeting these predictors in this intervention, sociocultural and emotive variables, such as anxiety and fatalism, were added as mediators of the relationship between awareness and actual preparedness behaviours, as well as that between awareness and personal responsibility (Joffe et al. 2013; Johnston et al. 2013; Paton 2008; Paton et al. 2010).

\subsection{Theoretical models and preparedness intervention studies}

In order to be effective, behaviour change interventions should be based on theoretical models that explain the behaviour change process (Michie et al. 2008). Various theoretical models have been used in natural hazard intervention studies, such as the person-relativeto-event model (Duval and Mulilis 1999), the precaution adoption process model (Weinstein and Sandman 1992) and the emotion-focused coping model (Lazarus and Folkman 1984). In the health field, the prediction of preparedness behaviours has been modelled according to the theory of planned behaviour and protection motivation theory (Abraham et al. 1998; Bennett and Murphy 1997). The inclusion of largely cognitive factors, as found in many of these models, is not sufficient for understanding the relationship between risk awareness and hazard preparedness. More holistic studies have attempted to develop models to predict the adoption of natural hazard preparedness with good success (Paton et al. 2003, 2005). 
Table 1 Study design chart

\begin{tabular}{llllll}
\hline $\begin{array}{l}\text { Groups } \\
(n=160)\end{array}$ & $\begin{array}{l}\text { Pre-survey } \\
\text { and checklist } \\
(1 \text { week } \\
\text { before } \\
\text { workshop) }\end{array}$ & Workshops & $\begin{array}{l}\text { Post-survey and } \\
\text { checklist } \\
(1 \text { week after the } \\
\text { end of } \\
\text { workshops })\end{array}$ & $\begin{array}{l}\text { Follow-up survey } \\
\text { and checklist } \\
(3 \text { months after the } \\
\text { end of } \\
\text { workshops })\end{array}$ & $\begin{array}{l}\text { Follow-up survey } \\
\text { and checklist } \\
(12 \text { months after } \\
\text { the end of } \\
\text { workshops })\end{array}$ \\
\hline $\begin{array}{c}\text { Intervention } \\
(n=80) \\
\begin{array}{c}\text { Control } \\
(n=80)\end{array}\end{array}$ & $\mathrm{X}$ & $\mathrm{X}$ & $\mathrm{X}(\$ 250)$ & $\mathrm{X}(\$ 40)$ & $\mathrm{X}(\$ 40)$ \\
\hline
\end{tabular}

Thus, the study reported in this paper utilised a modified version of Paton et al.'s model of natural hazard risk reduction preparedness (Paton et al. 2003, 2005), which proposes that motivation, outcome expectancy and self-efficacy beliefs are the main predictors of preparedness. Paton et al. add a fourth variable to their model: intentions to prepare. In addition, drawing from Paton et al.'s model, empowerment and social cohesion are added to the intervention as potential mediators of preparedness. In addition to drawing on Paton et al.'s model of preparedness, this study is also rooted in and draws on previous work on social representations of earthquakes in Seattle and Izmir (Joffe et al. 2013). Like the Paton et al. model, this work showed self-efficacy to be important, but added anxiety, trust, sense of responsibility and sociocultural variables such as fatalism versus sense of control as potential determinants of preparedness. Thus, two key models are drawn on in shaping this intervention with the former giving more emphasis to cognitive and empowerment variables and the latter to emotive and sociocultural factors.

This paper is divided into two parts. The first provides a detailed description of the methodology and design of the intervention study so that it can serve as a basis for the development of future community intervention studies. The second part describes results of the intervention conducted in Seattle in September 2015.

\section{Method and design}

\subsection{Study design and assessment periods}

This is a cross-cultural, quasi-experimental, non-randomised, controlled, longitudinal, face-to-face intervention study, with a pretest-posttest design. Study objectives are (1) to increase household preparedness for earthquakes and home fires in Seattle (USA) and Izmir (Turkey) by way of an intervention, (2) to evaluate changes in levels of critical awareness, self-efficacy, perceived outcome, trust, empowerment, anxiety and social cohesion, as well as levels of adjustment following the intervention, and (3) to compare results of the intervention across the different cultures. Participants in the intervention group each participate in two 3-h workshops (intervention), while controls do not receive the intervention. All participants complete assessments 1 week before and 1 week after the intervention, and they are followed up 3 and 12 months after the intervention to measure preparedness behaviour change (Table 1). 
Monetary incentives are given for participation in the study. In the study conducted in Seattle, ${ }^{1}$ participants in the intervention group were paid $\$ 250$ for completing baseline and post-intervention assessments, as well as a 6-h workshop divided over 2 days ( $3 \mathrm{~h}$ each day). Participants in the control group were given $\$ 100$ for completing the baseline and post-intervention assessments. To ensure completion of the pretest and posttest assessments, participants in both groups were paid only after completion of the second assessment. Participants are paid $\$ 40$ per completed follow-up assessment, at 3 and 12 months after the intervention. Study incentives for Izmir are equivalent to the monetary incentives in Seattle.

\subsection{Sample}

In order to define sample size, an a priori power analysis using G*Power statistical software, version 3.1 (Faul et al. 2009) was conducted. Equal-sized sample groups were planned, meaning that the allocation ratio of N1 to N2 is 1 (Bredenkamp 1969; Orme et al. 1990). To perform a two-group comparison of a primary outcome with a standard alpha of $0.05,64$ individuals per group, or a total of 128 individuals, provide $80 \%$ power to detect a significant difference of $5 \%$ between the intervention group and the control group in a two-tailed analysis. Consequently, we planned to recruit 100 participants per group to account for dropouts, in order to have a minimum of 64 participants per group left in the final longitudinal follow-up. The post-intervention sample in Seattle consisted of 157 people (85 intervention group and 72 control group), which decreased at 3 months to 145 (73 intervention group and 72 control group). ${ }^{2}$

The sample in Seattle consisted of adult residents (aged 18-80) of North Seattle, with a mean age of $50(\mathrm{SD}=13)$. Sixty per cent of residents were female, $77 \%$ identified as Caucasian, $47 \%$ identified as Christian, while $36 \%$ reported not having any religion; $59 \%$ were married or living with their partner and $75 \%$ were home owners. More than half of the sample reported being employed (52\%).

\subsection{Area selection}

Since sense of community and community participation have been found to be variables that affect preparedness and adoption of mitigation measures (Bishop et al. 2000; Paton 2000), intervening in an existing community seemed appropriate. Consequently, for the Seattle study, one area was selected for the intervention group and the intervention was conducted there. A geographically separate, equally large area served as the field for those in the control condition. In February 2015, the team of researchers travelled to Seattle to explore potential areas to conduct the study and ultimately selected two of them. ${ }^{3}$ Additional aims for the scoping trip included establishing contact with a recruitment agency and arranging meetings with Seattle emergency management experts, such as the Red Cross,

\footnotetext{
${ }^{1}$ The intervention in Seattle was conducted in September 2015, and the three-month follow-up assessment was conducted in January 2016. The intervention in Izmir was conducted between May and June of 2016.

${ }^{2}$ Eleven participants in the intervention group were living in subsidised housing, and thus, they were not able to make the required preparedness fixes at home. For this reason, their data were not included in the post-intervention and 3-month analysis. These data call for a separate analysis, to explore barriers to preparedness and the effect of the intervention on a vulnerable population.

${ }^{3}$ The radius from which participants were chosen was enlarged at the recruitment stage because the agency could not find sufficient numbers of people willing to partake, who fitted the criteria in the original, smaller communities sampled.
} 
the Seattle Fire Department and the Seattle Office for Emergency Management for information and collaboration in the intervention. This first trip to Seattle proved to be highly important in the design of the intervention and in making connections with experts and survivors of earthquakes, who agreed to serve as motivational speakers in the intervention. A similar trip was conducted for Izmir to explore and select the intervention areas as well as to establish contact with the respective emergency management authorities and trusted informants.

\subsection{Participant recruitment and allocation}

The sample constitutes adults (aged 18-80), one representative per household, who are residents of Seattle and Izmir. In each of these cities, the study areas constituted two geographically separated areas of equivalent demographic mix assigned to be the intervention and control groups, respectively. A recruitment agency from each city was hired to recruit participants. Area selection in the two cities was based on the same criteria.

In each of the two cities, the agency is instructed to recruit 100 participants from the intervention area and 100 from the control area. Specifically, they are asked, for both groups, to recruit evenly across each age group (18-35, 36-54, 55-80), 50\% male and $50 \%$ female within each age group, two-thirds home owners and one-third renters and $50 \%$ below $\$ 45,000$ and $50 \%$ above $\$ 45,000$ annual household income (and the equivalent respective salary in Izmir). All recruiting in Seattle was completed over the phone by the recruitment agency.

One week before the intervention in Seattle, participants were called by the agency to arrange a specific date and time to visit their homes to complete the baseline assessment. After completion, the intervention group participants were called and reminded of the day, date, time and location for their workshops. One week after completion of the workshops, participants in both groups were called by the agency to schedule a specific date and time to complete the post-workshop assessments and to receive their respective incentives for participation.

\subsection{Intervention design and delivery}

The intervention is named fix-it, as it focuses on fixing and securing items in the home. Fixit is a name that is easy to remember and has positive/neutral connotations, as opposed to threat and fear-inducing names with negative and catastrophic connotations. According to the literature on campaigns, in order to be effective in facilitating action, names and messages of preparedness campaigns should be positive in tone, promote a sense of empowerment, as well as being short, clear, consistent and motivating of action (Jones 2012; Kocher 1993). The intervention name received good feedback in the pilot study, with participants stating that the name was, "easy to remember", "fine", "concise and it implies action". ${ }^{4}$ Fix-it was also accepted as a good name for the intervention in Izmir, as a similar Turkish term exists.

Fix-it includes the key ingredients of previous preparedness interventions that have proven successful, such as hands-on training, face-to-face interactions, empowerment and

\footnotetext{
4 A pilot of this study was conducted with 11 residents of North-east Seattle in June 2015. The intervention proved to be effective in increasing preparedness behaviours in participants, with participants having adopted significantly more preparedness measures one week after the intervention, compared to their baseline levels of preparedness $(p=0.001)$.
} 
community cohesion. In addition, determinants of behaviour such as self-efficacy, outcome expectancy and motivation are targeted through different behavioural techniques (Michie et al. 2008). The intervention design was influenced by the work of Michie et al. (2011) concerning effective behaviour change techniques to challenge specific determinants of behaviour. To address motivation, the use of rewards, incentives (gift cards for group challenge winners, magnets and mugs with acronyms and the name of the intervention), social encouragement and support (engaging with the fix-it Facebook group page, taking pictures of their homes) were used, as was persuasive communication. Self-efficacy was addressed using self-monitoring (homework review), rehearsal (videogame play), coping skills learning, social encouragement and support and feedback (homework review); all behaviour change techniques have proven effective in changing self-efficacy (Michie et al. 2008). In order to affect outcome expectancy and increase motivation, persuasive communications were given by experts who were trusted sources, such as the American Red Cross, the Seattle Fire Department and the Seattle Office of Emergency Management (Michie et al. 2008). AFAD, the equivalent trusted source for emergency management in Turkey, and their experts were used as communicators in the Izmir intervention.

The intervention consisted of two 3-h interactive, face-to-face workshops for each participant in the active group and focused on securing items in the household. The workshops were led by two professionals, experts in emergency management training and psychologists. ${ }^{5}$ The workshops included hands-on training, as well as interactive tools, such as playing a video game, reviewing photographs of participants' homes and engaging in a Facebook group page. With the purpose of intervening in an existing community, the intervention was delivered in a community centre (as in the case of Seattle) or space where communities gather to participate in activities. The intervention was carefully designed by the psychology researchers of the project who, in addition to reviewing the literature on natural disaster preparedness intervention studies and that on behaviour change, consulted three experts in the field of interventions, epidemiological studies and behaviour change techniques to review the intervention. This, together with the pilot study conducted in June, shaped the fix-it intervention.

\subsubsection{Fix-it}

The 6-h fix-it workshop, divided over 2 days ( $3 \mathrm{~h}$ each), was delivered on weekdays after work. Specifically, it focused on securing nine items in the household (Table 2): securing bookcases and large cabinets, securing TVs and computer screens, making sure that sockets were not overcharged (e.g. not having a multi-plug plugged into another multiplug), having functioning smoke alarms and knowing how to test them, ensuring that no pictures or frames were hanging above sofas and/or beds, having all exits clear of obstruction and having in-date fire extinguishers. These nine items are taken from the 16 earthquake and 13 fire preparedness measures on questions 17 and 30 of the survey (Appendix I of supplementary material), and they constitute the primary outcome measure of preparedness (Table 2).

The literature on behaviour change interventions advocates that one limits interventions to few, simple behaviours to increase effectiveness (Michie et al. 2014). The fix-it intervention chose the nine items from a total of 29 preparedness items for earthquake and fire

\footnotetext{
5 The workshops were conducted with approximately 20 people in each. Therefore, four identical workshop groups were run on consecutive nights the first week, and then, people attended their second workshop one week later than their first.
} 
Table 2 Checklist of the nine fix-it preparedness measures

Please check whether each of the following 9 measures has been taken or not by the participant:

\begin{tabular}{|c|c|c|c|c|}
\hline & $\begin{array}{l}\text { No } \\
\text { (None) }\end{array}$ & $\begin{array}{l}\text { Yes } \\
\text { (Some) }\end{array}$ & $\begin{array}{l}\text { Yes } \\
\text { (All) }\end{array}$ & $\begin{array}{l}\text { Not } \\
\text { applicable }\end{array}$ \\
\hline 1. TV is secured (with Velcro, pad, straps, or locks) & $\bigcirc$ & $\bigcirc$ & $\bigcirc$ & $\bigcirc$ \\
\hline 2. Computer is secured (with Velcro, pad, straps, or locks) & $\bigcirc$ & $\bigcirc$ & $\bigcirc$ & $\bigcirc$ \\
\hline 3. Bookcase is secured to the wall (using nylon strap) & $\bigcirc$ & $\bigcirc$ & $\bigcirc$ & $\bigcirc$ \\
\hline 4. Large cabinet is secured to the wall (using nylon strap) & $\bigcirc$ & $\bigcirc$ & $\bigcirc$ & $\bigcirc$ \\
\hline $\begin{array}{l}\text { 5. No multiple plugs plugged in another multiple plug (Ask "Do } \\
\text { you have any multiple plug in use?" If so "Let me have a look at } \\
\text { them") Check NO if they have a multiple plug plugged into } \\
\text { another multiple plug }\end{array}$ & 0 & $\bigcirc$ & 0 & 0 \\
\hline $\begin{array}{l}\text { 6. No objects (framed pictures, mirrors) are placed above sofas } \\
\text { AND beds If posters without frame then OK; if posters WITH } \\
\text { frame click, "No" }\end{array}$ & $\bigcirc$ & $\bigcirc$ & O & \\
\hline 7. Exits (doors) are clear of obstruction & $\bigcirc$ & $\bigcirc$ & $\bigcirc$ & \\
\hline $\begin{array}{l}\text { 8. Functioning smoke alarms and respondent knows how to test } \\
\text { them ("Please, show me how to test it") All alarms have a test } \\
\text { button, even hardwired ones. If a person does not know how to } \\
\text { test them, then mark "No" }\end{array}$ & 0 & 0 & 0 & \\
\hline 9. Having an in-date fire extinguisher (if expired, then click "No") & 0 & 0 & 0 & \\
\hline
\end{tabular}

\footnotetext{
${ }^{a}$ Item removed from the analyses due to an error in instructions/interpretation and inconsistent scoring
}

that individuals could carry out at home with little cost and bureaucracy and without necessarily owning their own homes. This intervention did not include structural mitigation actions because the researchers aimed to target those behaviours over which people had control. These mitigative behaviours were also chosen, as opposed to focusing on promoting kits or plans, because of evidence that they are needed. According to the 2013 American Housing Survey (U.S. Census Bureau 2015), which added questions on emergency and disaster preparedness for the first time, $52 \%$ of American citizens reported having an emergency kit, $82 \%$ reported having non-perishable food stored, $62 \%$ stated having stored water and over $30 \%$ claimed having an emergency meeting location and a communication plan (Bureau 2015). Since having a kit and storing food and water had already been adopted by $60-80 \%$ of American residents, the fix-it intervention's focus was on improving other adjustment measures that are low cost and easy to adopt, such as securing items in the household, which were not measured in the American Housing Survey. In addition, securing items in the house has been identified as one of the basic categories of preparedness by victims of earthquakes, reducing injuries and allowing them to focus on other categories of preparedness, such as assisting others and following their emergency plans (Paton and Johnston 2015). Despite being considered as a key aspect of preparedness and its adoption being critical for people's outcomes in earthquakes, most preparedness studies focus on survival measures (e.g. storing food and water) and not on such mitigative behaviours (Russell et al. 1995; Spittal et al. 2006).

Day 1 of fix-it aimed to enhance the awareness and importance of being prepared. The workshop started with a free association task (Joffe and Elsey 2014), where participants were asked to write or draw their associations to "home" on a grid. This gauged how they represented the very homes that the intervention aimed to target and allowed them to share 
their thoughts and feelings about their homes and to begin to interact with others in their group. The grids were collected for content analysis by the researchers, with feedback provided at the next workshop. Participants were then presented with common cognitive and emotive responses regarding earthquakes that residents in each of these cities had held, as per findings of the previous study (Joffe et al. 2013), such as "denial of fear", or "fascination with earthquakes". A discussion was then held on how these might affect preparedness. Subsequently, a series of videos of previous earthquakes in their own city, survivor stories from each city and a home fire simulation were shown. Each of these videos was followed by a group discussion, where participants shared their experiences of earthquakes and fires and the impact that these had on them. Participants then engaged in playing "Beat the Quake", a videogame concerning earthquake and fire preparedness, in teams of five. To create social encouragement, promote group cohesion and give incentives, participants were then divided into groups where they participated in a group challenge based on a set of questions on earthquake and home fire preparedness. Before the end of the first day of the workshop, participants were given an assignment, to be reviewed on the second day of the workshop. The assignment involved taking pictures of the following items in their houses and sending them via email to the workshop leader: a smoke alarm, a multi-plug, an important exit, a bookshelf, a bed/and or couch, a fire extinguisher, a television and a computer. These pertained to the nine preparedness measures that $f i x$-it focused on. Participants were also invited to join a fix-it Facebook group page, with the purpose of creating a sense of community empowerment, feedback and an opportunity for social interaction with the researchers and the rest of the intervention participants, as well as for the sharing of learning. The Facebook group page was designed and managed by one of the researchers in the team.

Day 2 of the workshop focused on "how to" fix-it. The evening started with the feedback from participants' associations to home, followed by a discussion on the possibility of their homes being a space of danger in the event of an earthquake or a home fire. A review of their assignments followed, where photographs of the participants' homes were screened for discussion. This was followed by an examination of the nine preparedness measures with videos that explained to participants how to carry out the preparations. Trusted experts (in Seattle, the Red Cross for, the Seattle Fire Department and the Office of Emergency Management in the case of Seattle, AFAD in Izmir) then gave presentations and demonstrated live with tools how to secure items. Participants were invited to touch the earthquake preparedness and fire safety tools and interact with the experts by asking them questions at the "hands-on stations". At the end of the workshop, the prize for the winners of the group challenge was given, along with a fridge magnet with an acronym for the nine fix-it measures, a mug with the name of the intervention and a certificate of completion. These giveaways, as well as being a reward for having participated in the workshops, aimed to serve as reminders after the intervention was over, ensuring that the fix-it measures were adopted.

\subsubsection{Beat the Quake}

In order to ensure that participants learned, practiced and mastered the nine fix-it preparedness measures, they played a videogame (on individual tablets) at the end of the first day of the workshop. Beat the Quake was originally designed by Earthquake Country Alliance in 2006 as part of the Great Shake Out earthquake preparedness campaign in California. The game is free and easily accessible from its website (http://www. dropcoverholdon.org/beatthequake/game/). In the original version of the game, the player 
is asked to click on items in a virtual living room and correctly identify how to secure 14 of these items (including fix-it measures) with appropriate methods and tools. The player has to choose an answer from a list of options. The more items the player correctly secures, the higher the score received. The player has to act quickly before the big earthquake hits. The player receives audio, visual and textual feedback throughout the game and additional visual feedback at the end of the game. For the purpose of the fix-it intervention, Beat the Quake was modified with the help of UCL's EPICentre, by adding four fire preparedness items. A Turkish version also existed and was also modified to include the fire items. Beat the Quake was improved for the purpose of this study and is now a multi-hazard preparedness educational tool. Beat the Quake has proven to generate significant preparedness learning outcomes (Tanes and Cho 2013).

\subsection{Consent}

Ethical permission was obtained to conduct the study (UCL Ethics Project ID Number: 1392/001). Informed written consent was obtained from each participant before completing the baseline survey. A member of the recruitment agency collected all signed consent forms from participants in both groups. Both participants and recruitment agency members were blind to group allocation.

\subsection{Measures}

A 25-min computer-assisted survey and a checklist comprised the assessment that participants in both groups completed at the following time points in their homes ${ }^{6}$ : (1) at baseline, 1 week before the intervention, (2) 1 week after the intervention, (3) 3 months after and (4) 12 months after the intervention. At the first home visit, a member of the recruitment agency met with each participant and asked them to complete the baseline survey online. Once finished, the agency member proceeded with the second part of the assessment, which was to conduct a tour around the house and examine with a checklist whether the participant had or had not adopted each of the nine preparedness measures. This constituted the observational measure of the study. Home visits to observe and document participants' preparedness behaviours, rather than sole reliance on self-reports, offer a novel approach to this field.

\subsubsection{Survey}

A self-administered computer-assisted survey (see Appendix I of supplementary material) using Qualtrics software was used to assess the following variables:

- Demographics: Sociodemographic characteristics including gender, age, ethnicity, marital status, number of children, employment status, education level and annual income level.

- Building characteristics including information such as type of residence, year and material of construction of the residence and earthquake retrofitting.

- Barriers to preparedness for earthquake and for fire including a set of items that the person had to tick (all that apply) indicating barriers for not preparing, such as the lack

\footnotetext{
${ }^{6}$ The assessments were done on paper in Izmir as this was deemed more appropriate by the Turkish researchers employed on the project.
} 
of time, the financial cost, the need for cooperation with others, the skill required, information overload, none and other.

Precursor variables:

- Critical awareness: assessed by four questions that asked how often one thinks and talks about earthquakes and how often one thinks and talks about home fires, with responses on a Likert-type scale from 1 (never) to 5 (once a week or more). This is a modified version of Dalton's measure of critical awareness (Dalton et al. 2001) that has been used in other studies with good success (Paton et al. 2003)

- Earthquake anxiety: measured by a set of items (e.g. "I avoid thinking about earthquakes") on a Likert-type scale ranging from 1 (not at all) to 4 (a great deal). It was developed by Johnston (Paton et al. 2003) and used in several earthquake preparedness studies by Paton.

- Self-efficacy: used a modified version of Riggs and Knight's scale (Riggs and Knight 1994).

- Intention to prepare was assessed by the question "Do you intend to do it in the next week or two?" for each of the nine preparedness measures.

- Outcome expectancy: The perception of whether personal actions would effectively mitigate or reduce the threat was assessed by a set of five questions for each of the nine preparedness measures, to which respondents could answer yes or no.

Mediators:

- Trust: measured by asking the participant how much trust they had in several institutions including education, armed forces, government, scientists and the church, rated from 1 (none at all) to 4 (a great deal) (Joffe et al. 2013).

- Fatalism: evaluated by a set of questions used in a previous study (Joffe et al. 2013)

- Empowerment: used a modified version of Speer and Peterson's empowerment scale (Speer and Peterson 2000).

- Social cohesion: used a reduced version of the scale used to measure social cohesion in European countries (Dickes and Valentova 2013).

- Collective efficacy: used Riggs and Knight's scale version in modified form (Riggs and Knight 1994).

- Sense of responsibility: assessed by the question "how much responsibility do the following people and institutions have for reducing your risk from earthquakes?" The same question was asked for home fires (Joffe et al. 2013).

- Perceived level of corruption of the government and the construction industry was assessed by asking: "On a scale from 1 (None at all) to 4 (A great deal) how would you rate the level of corruption of the following: the local government, the state government, the federal government, the construction industry?" (Joffe et al. 2013)

- Religiousness: was assessed using a single question ("How religious would you say you are?") rated on a five-point scale, ranging from "not at all" to "very much".

Outcome variables:

- Earthquake preparedness was assessed by a set of 16 earthquake preparedness items, which included the five earthquake-related fix-it measures; participants had to indicate whether they had adopted them or not. This set of items used to assess preparedness is a modified version of the set of items used in a previous study (Joffe et al. 2013).

- Fire preparedness was measured by a set of 13 fire preparedness items, including the four fix-it measures that gauged fire preparedness. 


\subsubsection{Checklist}

The nine-item checklist constitutes the primary outcome measure (Table 2). As described above, a member of the recruitment agency examined the participant's household and checked whether each of the nine preparedness measures had been adopted by the participant or not. This is a purely observational measure, and it was evaluated alongside the self-report measure.

\subsubsection{Follow-ups}

Follow-up assessments included a modified version of the survey (with additional questions about the workshop) and the checklist. Follow-up assessments were conducted 3 and 12 months after the end of the workshops.

\subsection{Interrater reliability}

Interrater reliability on $5 \%$ of the checklists in all countries, using two independent raters, produced a satisfactory result (kappa $=0.65)$.

\subsection{Data analysis and management}

The SPSS statistical software package, version 20 (Nie et al. 2011), was used for conducting the statistical analysis. Behaviour change was measured in both groups, and tests were conducted to evaluate differences between means of behaviour change between groups. Logistic regression analysis of the outcome measure on the active group was conducted, with adjusted confidence intervals and $p$ values. In addition, cross-tabulation and $\chi^{2}$ statistics were carried out to evaluate correlations between self-report and observational preparedness measures to determine the extent of agreement between them. Moderated mediation analysis among relevant variables was carried out to explore mediators of preparedness. Levels of anxiety in relation to preparedness behaviours were also examined. Although preparedness is measured as an overall score, including earthquake and fire measures, separate analyses for earthquake and home fire preparedness were conducted in order to explore differences in preparedness for the more every day risk (i.e. fires) versus the more long return period risk (i.e. earthquakes). It must be noted that it is the difference in scores between assessment times that is the key outcome: that is, the study measures behaviour change. ${ }^{7}$

\section{Results: Seattle study}

\subsection{Baseline analyses (Tables 3, 4, 5, 6)}

The sample in Seattle was adult residents (aged 18-80) of North Seattle, with a mean age of $50(\mathrm{SD}=13)$. Sixty per cent of residents were female, $77 \%$ identified as Caucasian,

\footnotetext{
7 If an item is not applicable at both time points, then there is no change. However, if a participant goes from not applicable to showing the behaviour, then there is behaviour change because some act has taken place. For example, if a person did not have a TV at time 1, but had a TV at time 2, which they had secured, then they had carried out a positive act (securing the TV) (see Appendix II of supplementary material).
} 
Table 3 Preparedness levels at baseline

\begin{tabular}{|c|c|c|c|c|c|c|}
\hline & \multicolumn{2}{|c|}{$\begin{array}{l}\text { Intervention } \\
(n=85)\end{array}$} & \multicolumn{2}{|c|}{$\begin{array}{l}\text { Control } \\
(n=72)\end{array}$} & \multicolumn{2}{|c|}{$\begin{array}{l}\text { Total } \\
(n=157)\end{array}$} \\
\hline & M & SD & M & SD & M & SD \\
\hline Overall preparedness & 13.32 & 2.76 & 13.32 & 2.51 & 13.32 & 2.64 \\
\hline Earthquake preparedness & 6.11 & 2.22 & 5.68 & 1.89 & 5.91 & 2.08 \\
\hline Fire preparedness & 7.21 & 1.51 & 7.64 & 1.46 & 7.41 & 1.50 \\
\hline
\end{tabular}

Table 4 Barriers for preparedness at baseline

\begin{tabular}{llllll}
\hline Barriers for earthquake preparedness & Mean & SD & Barriers for fire preparedness & Mean & SD \\
\hline Other things/problems to think about & 0.49 & 0.50 & Other things/problems to think about & 0.49 & 0.50 \\
Time required to do it & 0.40 & 0.49 & Skill or knowledge required & 0.28 & 0.45 \\
Skill or knowledge required & 0.30 & 0.45 & Time required to do it & 0.24 & 0.43 \\
The financial cost & 0.26 & 0.44 & Need for cooperation with others & 0.16 & 0.36 \\
Need for cooperation with others & 0.20 & 0.40 & The financial cost & 0.11 & 0.31 \\
Information overload & 0.14 & 0.34 & Information overload & 0.10 & 0.29 \\
\hline
\end{tabular}

$47 \%$ as Christian, while $36 \%$ reported not having any religion; $59 \%$ were married or living with their partner; and $75 \%$ were home owners. More than half of the sample reported being employed (52\%). Baseline differences in sociodemographic, psychosocial and preparedness characteristics of participants were assessed before the intervention in order to rule out selection bias due to the non-randomised sampling design of the study. There were no differences in sociodemographic features or psychological variables between the intervention and control groups at baseline. In addition, no significant differences were found between the intervention and control groups in terms of their preparedness levels (Table 3).

Both the intervention and control groups reported barriers to earthquake and fire preparedness. The most common barrier reported was "other things/problems to think about", followed by "the time required to do it" and "the skill or knowledge required". The least reported barrier for preparing for earthquakes was "the financial cost" (Table 4).

Predictors of preparedness before the intervention are listed in Table 5. It is important to note that all results are given first for overall preparedness, which refers to an average of the five earthquake and the three fire preparedness items. ${ }^{8}$ Results show that empowerment $\left(F(1,154)=9.1 ; p=0.003, R^{2}=0.05\right)$, trust $\left(F(1,154)=4.0 ; p=0.046, R^{2}=0.02\right)$, corruption $\left(F(1,155)=6.2 ; p=0.01, R^{2}=0.03\right)$ and social cohesion $(F(1,155)=1.6$; $\left.p=0.04, R^{2}=0.23\right)$ predicted overall preparedness in a univariate regression analysis at baseline. Predictors of earthquake preparedness, in particular, at baseline were empowerment $\left(F(1,154)=4.5 ; p=0.034, R^{2}=0.02\right)$, trust $\left(F(1,154)=3.9 ; p=0.04, R^{2}=0.02\right)$,

\footnotetext{
${ }^{8}$ The item of safe multi-plugs was removed from the analyses due to an error in instructions and inconsistent scoring. Thus, analyses included eight preparedness items, five checklist items for earthquake and three for fire preparedness.
} 


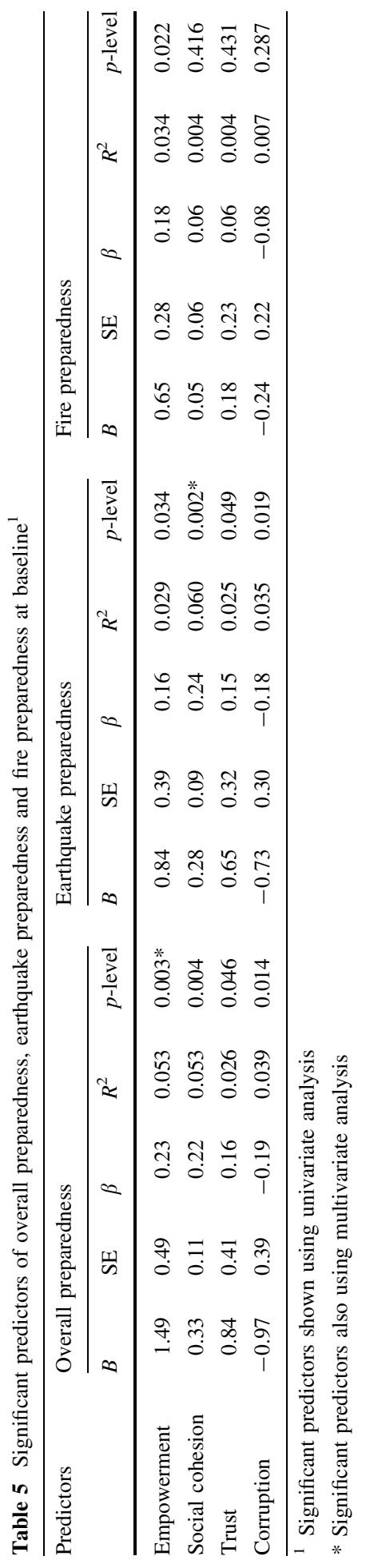


Table 6 Correlations between self-report and observational measures of earthquake and fire preparedness

\begin{tabular}{|c|c|c|c|c|c|c|c|c|}
\hline & $\begin{array}{l}\text { TV } \\
\text { secured }\end{array}$ & $\begin{array}{l}\text { Computer } \\
\text { secured }\end{array}$ & $\begin{array}{l}\text { Bookcase } \\
\text { secured }\end{array}$ & $\begin{array}{l}\text { Large } \\
\text { cabinet } \\
\text { secured }\end{array}$ & $\begin{array}{l}\text { No objects } \\
\text { above } \\
\text { sofas/beds }\end{array}$ & $\begin{array}{l}\text { Exits } \\
\text { clear }\end{array}$ & $\begin{array}{l}\text { Fire } \\
\text { extinguisher }\end{array}$ & $\begin{array}{l}\text { Smoke } \\
\text { alarm }\end{array}$ \\
\hline TV secured & $0.333 * *$ & & & & & & & \\
\hline $\begin{array}{r}\text { Computer } \\
\text { secured }\end{array}$ & & 0.010 & & & & & & \\
\hline $\begin{array}{r}\text { Bookcase } \\
\text { secured }\end{array}$ & & & $0.601 * *$ & & & & & \\
\hline $\begin{array}{l}\text { Large cabinet } \\
\text { secured }\end{array}$ & & & & $0.438 * *$ & & & & \\
\hline $\begin{array}{l}\text { No objects above } \\
\text { sofas/beds }\end{array}$ & & & & & -0.122 & & & \\
\hline Exits clear & & & & & & 0.034 & & \\
\hline Fire extinguisher & & & & & & & $0.557 * *$ & \\
\hline Smoke alarm & & & & & & & & $0.198^{*}$ \\
\hline
\end{tabular}

corruption $\left(F(1,155)=5.6 ; p=0.01, R^{2}=0.03\right)$ and social cohesion $(F(1,155)=9.8$; $\left.p=0.00 ; R^{2}=0.05\right)$. Empowerment was the only predictor for fire preparedness at baseline.

Correlations between self-reported preparedness in the survey and observational preparedness measures in the checklist are described in Table 6. All items correlated significantly with each other except "computer is secured", "all exits are clear of obstruction" and "no objects are hanging above sofas/beds".

\subsection{Changes in preparedness levels after the intervention (Table 7; Figs. 1, 2,3)}

One week post-intervention, the intervention group had significantly improved its overall preparedness $(t(72)=3.8, p<0.001)$, as well as its earthquake $(t(72)=2.4, p=0.018)$ and fire preparedness $(t(72)=3.7, p<0.001)$ compared to baseline. Those in the control group improved their overall preparedness compared to baseline, though not significantly. The control group's levels of earthquake preparedness decreased slightly though not significantly from baseline, but they had significantly increased their fire preparedness $(t(71)=2.4, p=0.019)$. Between-group differences of preparedness change means 1 week after the intervention were significant for overall preparedness change $(p=0.001)$ and for earthquake preparedness change $(p=0.019)$, with the intervention group showing significantly more change (Table 7).

Three months after the intervention, both the intervention $(t(66)=3.3, p=0.001)$ and control groups $(t(67)=2.2, p=0.032)$ had significantly improved their overall preparedness levels compared to baseline. Although both groups had improved their earthquake preparedness 3 months after the intervention, the scores did not reach statistical significance. Fire preparedness improved for both groups at 3-month follow-up compared to baseline, but only the intervention group's fire preparedness reached statistical significance $(t(66)=5.6, p<0.001)$ (Table 7).

Multivariate analysis of variance was conducted to explore levels of preparedness change between groups across the two points in time, 1 week after the intervention and at the 
Table 7 Changes in preparedness levels 1 week after the intervention and at 3-month follow-up

\begin{tabular}{|c|c|c|c|c|c|c|c|c|c|c|}
\hline \multirow[t]{2}{*}{ Post-intervention $^{\mathrm{a}}$} & \multicolumn{4}{|c|}{$\begin{array}{l}\text { Intervention } \\
(n=73)\end{array}$} & \multicolumn{4}{|c|}{$\begin{array}{l}\text { Control } \\
(n=72)\end{array}$} & \multicolumn{2}{|c|}{$\begin{array}{l}\text { Between } \\
\text { groups }\end{array}$} \\
\hline & M & $\mathrm{SD}$ & $t$ test & $p$-level & $M$ & SD & $t$ test & $p$-level & $t$ test & $p$-level \\
\hline $\begin{array}{l}\text { Overall preparedness } \\
\text { change }\end{array}$ & 1.48 & 3.30 & 3.83 & $<0.001$ & 0.18 & 2.90 & 0.52 & 0.60 & 2.51 & 0.013 \\
\hline $\begin{array}{l}\text { Earthquake } \\
\text { preparedness change }\end{array}$ & 0.85 & 2.99 & 2.42 & 0.018 & -0.22 & 2.42 & -0.77 & 0.40 & 2.36 & 0.019 \\
\hline $\begin{array}{l}\text { Fire preparedness } \\
\text { change }\end{array}$ & 0.62 & 1.44 & 3.65 & $<0.001$ & 0.38 & 1.32 & 2.39 & 0.019 & 1.05 & 0.296 \\
\hline \multicolumn{11}{|l|}{ 3-month follow-up } \\
\hline $\begin{array}{l}\text { Overall preparedness } \\
\text { change }\end{array}$ & 0.93 & 2.28 & 3.31 & 0.001 & 0.91 & 3.44 & 2.18 & 0.032 & 0.27 & 0.978 \\
\hline $\begin{array}{l}\text { Earthquake } \\
\text { preparedness change }\end{array}$ & 0.07 & 1.98 & 0.30 & 0.80 & 0.47 & 2.82 & 1.37 & 0.20 & -0.94 & 0.348 \\
\hline $\begin{array}{l}\text { Fire preparedness } \\
\text { change }\end{array}$ & 0.88 & 1.29 & 5.55 & $<0.001$ & 0.38 & 1.65 & 1.90 & 0.061 & 1.94 & 0.054 \\
\hline
\end{tabular}

${ }^{a}$ One week after the end of the intervention

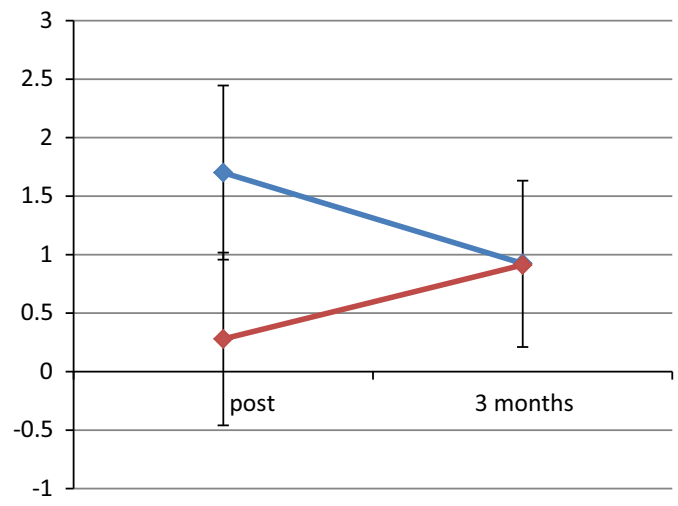

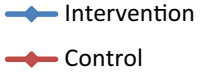

95\% confidence intervals shown

Fig. 1 Overall preparedness change 1 week and 3 months after the intervention

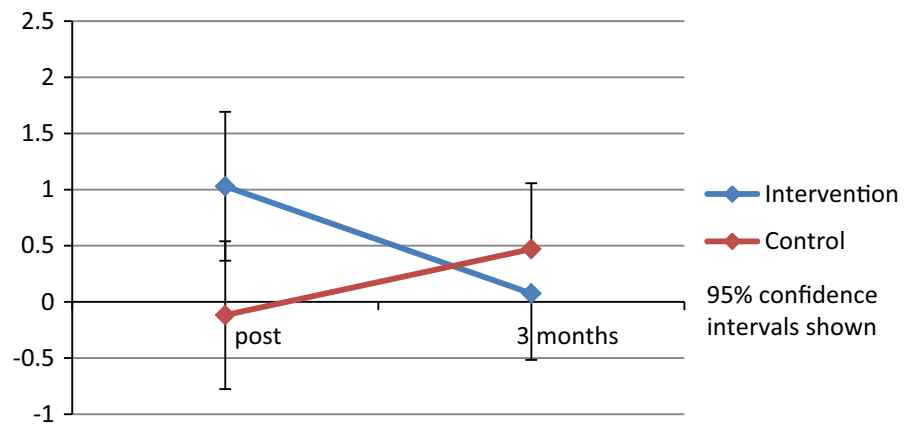

Fig. 2 Earthquake preparedness change 1 week and 3 months after the intervention 


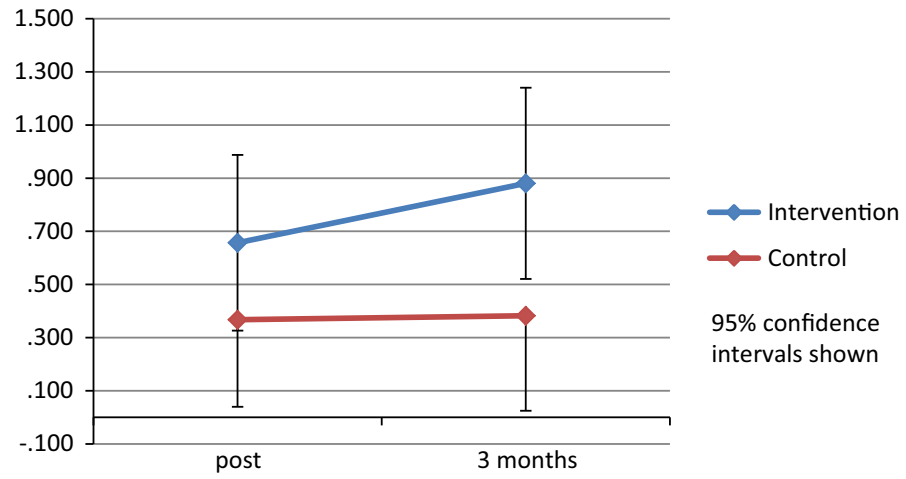

Fig. 3 Fire preparedness change 1 week and 3 months after the intervention

3-month follow-up. Results show a significant interaction of time and group ( $p=0.013)$, with the intervention group significantly decreasing their overall preparedness from time 1 (post-intervention) to time 2 (3-month follow-up) compared to the control group (Fig. 1). A similar pattern can be found regarding earthquake preparedness $(p=0.002)$ (Fig. 2). There was no significant interaction for group or time in fire preparedness between the two time points (Fig. 3).

\subsection{Predictors of preparedness change (Table 8)}

No significant predictors of changes in overall preparedness, earthquake or fire preparedness were found for the intervention group at 3-month follow-up using both univariate and multivariate analysis. However, for the control group, anxiety $(F(9,58)=3.5$; $p=0.011, R^{2}=0.355$ ) and trust in different systems (e.g. education, armed forces,

Table 8 Significant predictors of preparedness change at 3-month follow-up ${ }^{1}$

\begin{tabular}{|c|c|c|c|c|c|c|c|c|c|}
\hline & \multicolumn{4}{|c|}{$\begin{array}{l}\text { Intervention } \\
(n=73)\end{array}$} & \multicolumn{5}{|c|}{$\begin{array}{l}\text { Control } \\
(n=72)\end{array}$} \\
\hline & B & SE & $\beta$ & $\mathrm{R}^{2}$ & $\mathrm{~B}$ & SE & $\beta$ & $\mathrm{R}^{2}$ & $p$-level \\
\hline \multicolumn{10}{|c|}{ Predictors overall preparedness } \\
\hline Self-efficacy & 0.14 & 0.71 & 0.29 & 0.097 & 1.78 & 0.84 & 0.25 & 0.355 & 0.039 \\
\hline Collective efficacy & -0.23 & 0.70 & -0.04 & 0.097 & -1.93 & 0.92 & -0.23 & 0.355 & 0.041 \\
\hline Anxiety (earthquake) & 1.01 & 0.93 & 0.16 & 0.097 & 2.35 & 0.90 & 0.30 & 0.355 & $0.011 *$ \\
\hline Trust & 0.10 & 0.64 & 0.02 & 0.097 & -1.35 & 0.60 & -0.25 & 0.355 & $0.030 *$ \\
\hline \multicolumn{10}{|c|}{ Predictors of earthquake preparedness } \\
\hline Anxiety (earthquake) & 0.65 & 0.77 & 0.12 & 0.166 & 1.60 & 0.77 & 0.24 & 0.297 & 0.043 \\
\hline Trust & -0.12 & 0.54 & -0.03 & 0.166 & -1.40 & 0.52 & -0.32 & 0.297 & 0.009 \\
\hline \multicolumn{10}{|c|}{ Predictors of fire preparedness } \\
\hline Self-efficacy & -0.09 & 0.41 & -0.03 & 0.069 & 0.93 & 0.45 & 0.27 & 0.188 & 0.045 \\
\hline
\end{tabular}

1 Results of multivariate analysis reported

* Significant predictors with univariate analysis 
government, scientists and church) $\left(F(9,58)=3.5 p=0.030, R^{2}=0.135\right)$ were found to be predictors of overall preparedness at 3 months, both using univariate and multivariate analysis (multivariate results are presented in Table 8). In addition, using multivariate analysis (including the variables of self-efficacy, outcome expectancy, fatalism, collective efficacy, anxiety, empowerment, trust, corruption and social cohesion), self-efficacy $(p=0.03)$ and collective efficacy $(p=0.04)$ were significant predictors of overall preparedness for the control group (Table 8).

In terms of the specific predictors of earthquake and fire preparedness change at the 3-month follow-up, only anxiety $\left(F(9,58)=2.7 ; p=0.043, R^{2}=0.297\right)$ and trust $\left(F(9,58)=2.7 ; p=0.009, R^{2}=0.297\right)$ were significant predictors of earthquake preparedness, for the control group only (within a multivariate analysis of self-efficacy, outcome expectancy, fatalism, collective efficacy, anxiety, trust, corruption, empowerment and social cohesion). The only predictor for fire preparedness change at 2 months was selfefficacy $\left(F(9,58)=1.49 ; p=0.045, R^{2}=0.188\right)$, and this was only for the control group.

\section{Discussion}

This cross-cultural, longitudinal, community-based intervention aims to improve earthquake and home fire preparedness at the household level. The intervention's theoretical background, methodology, content and assessment process are thoroughly described above. In addition, results from the Seattle study and the 3-month follow-up are presented. Such detailed methodological descriptions, as well as the combination of self-report and observational measures to assess preparedness, are extremely rare in the natural disaster intervention literature. Therefore, this protocol seeks to provide a guideline for developing future, empirically based natural disaster preparedness interventions.

One week after the intervention, the intervention group had significantly improved its overall preparedness levels, as well as its earthquake and fire preparedness levels, showing the effect of the fix-it workshop on behaviour change in the intervention group. The control group, on the other hand, did not increase overall preparedness significantly: earthquake preparedness decreased slightly but fire preparedness increased significantly.

However, 3 months after the intervention, both groups had significantly improved their overall preparedness levels compared to baseline. Earthquake preparedness improved for both groups at the 3-month follow-up compared to baseline levels, though not significantly, while fire preparedness improved for both groups at the 3-month follow-up compared to baseline, but only significantly in the intervention group. Overall, results show the effect of the fix-it workshop on behaviour change in the intervention group, significantly improving their preparedness levels both 1 week after and 3 months after the intervention. More surprisingly, preparedness levels for the control group also increased significantly at 3 months. The control group caught up with the intervention group, who showed an unsurprising drop off compared to 1 week after the intervention. This leaves both groups significantly above baseline at 3 months, but not different from each other. Since controls did not receive the fix-it intervention, the home visits, which were conducted face-to-face and included a survey and observation of home earthquake and fire preparedness, seem to have played a role in subsequently increasing preparedness behaviour, particularly regarding earthquakes, in what had originally been designed as a control group.

The novel approach of combining self-report and observational measures of preparedness also proved to be of value. Correlation between self-report and observational 
data was good but not excellent, suggesting that there was variation in how items were observed and showing that results from self-report and observation differ. This study cannot determine which one is the more ecologically valid measure. Future studies would need to refine this.

Corroborating both the Paton et al. (2003, 2005) and Joffe et al. (2013) models, baseline results showed that overall preparedness is predicted by feeling empowered and by living in a socially cohesive community, as well as by having higher levels of trust in the systems that surround one. Perhaps more surprising is that representations of one's government being somewhat corrupt predict preparedness. Overall, the findings suggest that empowered, cohesive communities, who have trust in their social systems (e.g. education, scientists, the church and health) and who come from an individualistic culture, such as the USA, might put more effort into self-preparation, rather than relying on "corrupt" governmental authorities to protect them.

Another important finding is the significant role that emotions play in this study. Consistent with the existing literature, anxiety was found to have a direct effect on preparedness behaviours (Lee and Lemyre 2009; Miceli et al. 2008; Paton et al. 2003; Rüstemli and Karanci 1999) but only for the control group. Despite some research suggesting that high anxiety can hinder preparedness, this study suggests that the home visits may have produced an optimum level of anxiety, since the literature indicates that optimum levels are needed to bring about preparedness behaviours. Anxiety levels for the control group increased over time, compared to the intervention group, causing participants to increase their preparedness behaviour. It is likely that their anxiety increased due to having home assessments and yet not being given information on preparedness, as opposed to the intervention group, who received a 6-h workshop over two days on exactly what to do to be better prepared and whose levels of anxiety decreased after the intervention.

Trust in various systems (e.g. education, police, government, church and scientists) was another emotion that directly predicted preparedness only for the control group. This is consistent with previous research that shows that trust is a determinant of community action (Dillon and Phillips 2001). Other studies have found trust to moderate the relationship between intention to prepare and actual preparedness (Paton et al. 2003) (JohnsonGeorge and Swap 1982; Paton 2007; Sjoberg 1999). In this study, since the control group had no information about how to prepare as they did not attend the workshops, trust in authorities may have increased over time as a way of coping with their worry about not knowing what to do to become better prepared.

The possibility that the home visits acted as an intervention could have been determined by the inclusion of a second control group, who would not have undergone the baseline assessment. This possibility was considered, but the very assessment process in this study, done at whatever time point, potentially creates change and so this option was not seen as useful. Another potential limitation was that participants were not randomly assigned to the intervention versus control groups, as the researchers purposively sought to run the intervention in particular communities and to draw the control group from equivalent but separate communities. Despite non-random assignment, results showed no differences between groups on sociodemographic, psychosocial and preparedness variables at baseline.

Overall, it seems that the very act of going into people's houses, interacting with them face-to-face and having someone check their preparedness levels, as well as their emotions and cognitions, produced behaviour change, in this case an increase in overall, earthquake and fire preparedness over time. When people are left with anxiety, concerning what they should be doing to improve their preparedness and are having their preparedness levels 
gauged at predetermined intervals by an observer as well as via self-report, they may seek to engage in preparedness measures and may thereby improve their preparedness.

This study constitutes one of the first to evaluate both earthquake and home fire preparedness and that aims to compare results across two different cultures: Seattle (USA) and Izmir (Turkey). ${ }^{9}$ In addition, this multi-hazard preparedness intervention study uses both self-report and observational measures. Furthermore, the intervention was carefully designed by a team of multidisciplinary researchers, from the fields of psychology and engineering, who had previously evaluated the social representations of earthquakes in the people of Seattle and Izmir (Joffe et al. 2013). Results of these thorough interviews have allowed this team to develop detailed interventions tailored to match the social representations of each location (Seattle and Izmir). The researchers approached and designed both the survey and the intervention with depth knowledge of how people in the two cities conceptualise earthquakes. That study had highlighted the potential role played by emotive and sociocultural factors, rather than purely cognitive factors, in shaping earthquakerelated conceptualisations and behaviours. This sensitised the researchers to ensure that the current intervention study should carefully assess emotions such as anxiety and trust. They were, indeed, found to be critical in increasing the adoption of preparedness behaviours, though only in the control group. The sociocultural elements identified in the previous study can only be fully assessed once the Seattle component of the intervention is compared with the results of the Izmir intervention.

This protocol aims to improve the field of hazard preparedness, being one of the few papers that fully describe a rigorous methodology and rationale for a natural hazards intervention. This study has significant implications for the field of natural disaster preparedness at an international level, for earthquake and fire preparedness more specifically and, in particular, for the two cities studied, since it allows for replication and therefore improvement of intervention studies. Furthermore, in terms of impact, the present study suggests that earthquake and fire preparedness can be increased significantly by going into people's homes to observe whether they have prepared for earthquakes and fires at multiple, prearranged time points, as well as surveying their psychosocial characteristics relevant to preparedness, without any further intervention. This face-to-face element may well contain an ingredient that precipitates change in a way that digital methods do not.

\section{Governance and ethical considerations}

The research was funded by the Engineering and Physical Science Research Council, UK (Grant ref. EP/F012179/1). There are no known conflicts of interest. Ethical permission was obtained to conduct the study: UCL Ethics Project ID Number: 1392/001. Participants all signed an informed consent form before the study commenced.

Open Access This article is distributed under the terms of the Creative Commons Attribution 4.0 International License (http://creativecommons.org/licenses/by/4.0/), which permits unrestricted use, distribution, and reproduction in any medium, provided you give appropriate credit to the original author(s) and the source, provide a link to the Creative Commons license, and indicate if changes were made.

\footnotetext{
${ }^{9}$ Results from Izmir and longitudinal (1-year follow-up) results from Seattle will be presented in subsequent papers.
} 


\section{References}

Abraham C, Sheeran P, Johnston M (1998) From health beliefs to self-regulation: theoretical advances in the psychology of action control. Psychol Health 13:569-591

American Housing Survey for the United States: 2013 (2015) US census bureau. http://www.census.gov/ programs-surveys/ahs.html

Ballantyne M, Paton D, Johnston D, Kozuch M, Daly M (2000) Information on volcanic and earthquake hazards: the impact on awareness and preparation. Institute of Geological and Nuclear Sciences Limited Science Report Wellington

Bennett P, Murphy S (1997) Psychology and health promotion. Open University Press, Buckingham

Bishop B, Paton D, Syme G, Nancarrow B (2000) Coping with environmental degradation: salination as a community stressor. Network 12:1-15

Bredenkamp J (1969) The application of significance tests in theory-testing experiments. Psychol Beitr 11(3):275-285

Chan J, To H, Chan E (2006) Reconsidering social cohesion: developing a definition and analytical framework for empirical research. Soc Indic Res 75:273-302

CRED, UNISDR (2015) The human cost of weather-related disasters, 1995-2015. United Nations, Geneva

Dalton JH, Elias MJ, Wandersman A (2001) Community psychology. Wadsworth, Belmont

Dickes P, Valentova M (2013) Construction, validation and application of the measurement of social cohesion in 47 European countries and regions. Soc Indic Res 113:827-846

Dillon J, Phillips M (2001) Social capital discussion paper. Unpublished manuscript, Curtin University, Perth, Western Australia

Duval T, Mulilis JP (1999) A person-relative-to-event (PrE) approach to negative threat appeals and earthquake preparedness: a field study 1. J Appl Soc Psychol 29:495-516

Eisenman DP, Glik D, Gonzalez L, Maranon R, Zhou Q, Tseng CH, Asch SM (2009) Improving Latino disaster preparedness using social networks. Am J Prev Med 37:512-517

Faul F, Erdfelder E, Buchner A, Lang AG (2009) Statistical power analyses using G* power 3.1: tests for correlation and regression analyses. Behav Res Methods 41:1149-1160

Glik DC, Eisenman DP, Zhou Q, Tseng CH, Asch SM (2014) Using the precaution adoption process model to describe a disaster preparedness intervention among low-income Latinos. Health Educ Res 29:272-283

Joffe H, Elsey J (2014) Free association in psychology and the grid elaboration method. Rev Gen Psychol $18: 173$

Joffe H, Rossetto T, Solberg C, O’Connor C (2013) Social representations of earthquakes: a study of people living in three highly seismic areas. Earthq Spectra 29:367-397

Johnson-George C, Swap WC (1982) Measurement of specific interpersonal trust: construction and validation of a scale to assess trust in a specific other. J Pers Soc Psychol 43:1306

Johnston D et al (2013) Community understanding of, and preparedness for, earthquake and Tsunami Risk in Wellington, New Zealand. In: Joffe H, Rossetto T, Adams J (eds) Cities at risk, vol 33. Advances in natural and technological hazards research. Springer Netherlands, pp 131-148. doi:10.1007/978-94007-6184-1_8

Jones AM (2012) Use of fear and threat-based messages to motivate preparedness: costs, consequences and other choices: part one. J Bus Contin Emerg Plan 6:180-191

Karanci N, Aksit B (2000) Building disaster-resistant communities: lessons learned from past earthquakes in Turkey and suggestions for the future. Int J Mass Emerg Disasters 18:403-416

Karanci AN, Aksit B, Dirik G (2005) Impact of a community disaster awareness training program in Turkey: does it influence hazard-related cognitions and preparedness behaviors. Soc Behav Personal Int J 33:243-258

Kocher KW (1993) The stop AIDS story, 1987-1992. Swiss Aids Foundation and the Federal Office of Public Health, Switzerland

Lazarus RS, Folkman S (1984) Stress, appraisal, and coping. Springer, New York

Lee JE, Lemyre L (2009) A social-cognitive perspective of terrorism risk perception and individual response in Canada. Risk Anal 29:1265-1280

Lindell M (2013) North American cities at risk: household responses to environmental hazards. In: Rossetto T, Joffe H, Adams J (eds) Cities at risk. Springer, Netherlands, pp 109-130

Lindell MK, Whitney DJ (2000) Correlates of household seismic hazard adjustment adoption. Risk Anal 20:13-26. doi:10.1111/0272-4332.00002

Maskrey A, Safaie S (2015) GAR global risk assessment. In: EGU general assembly conference abstracts, p 6494 
McGee TK, Russell S (2003) "It's just a natural way of life..." an investigation of wildfire preparedness in rural Australia Global Environmental Change Part B. Environ Hazards 5:1-12

Miceli R, Sotgiu I, Settanni M (2008) Disaster preparedness and perception of flood risk: a study in an alpine valley in Italy. J Environ Psychol 28:164-173. doi:10.1016/j.jenvp.2007.10.006

Michie S, Johnston M, Francis J, Hardeman W, Eccles M (2008) From theory to intervention: mapping theoretically derived behavioural determinants to behaviour change techniques. Appl Psychol 57:660-680

Michie S, Van Stralen MM, West R (2011) The behaviour change wheel: a new method for characterising and designing behaviour change interventions. Implement Sci 6:42

Michie S, Atkins L, West R (2014) The behaviour change wheel: a guide to designing Interventions. Silverback, UK

Miller TR, Bergen G, Ballesteros MF, Bhattacharya S, Gielen AC, Sheppard MS (2014) Increasing smoke alarm operability through theory-based health education: a randomised trial. J Epidemiol Community Health 68:1168-1174

Nie N, Hull C, Bent D (2011) IBM statistical package for the social sciences (SPSS version 20) computer software. Chicago, SPSS

Orme JG, Borenstein M, Cohen J (1990) Statistical power analysis: a computer program. Soc Serv Rev 64(3):518-520

Paton D (2000) Emergency planning: integrating community development, community resilience and hazard mitigation. J Am Soc Prof Emerg Manag 7:109-118

Paton D (2007) Preparing for natural hazards: the role of community trust. Disaster Prev Manag Int J 16:370-379

Paton D (2008) Risk communication and natural hazard mitigation: how trust influences its effectiveness. Int J Glob Environ Issues 8:2-16

Paton D, Johnston DM (2015) The Christchurch earthquake: integrating perspectives from diverse disciplines. Int J Disaster Risk Reduc 14:1-5

Paton D, Smith LM, Johnston DM (2000) Volcanic hazards: risk perception and preparedness. N Z J Psychol 29:86-91

Paton D, Smith L, Johnston D, Johnston M, Ronan K (2003) Developing a model to predict the adoption of natural hazard risk reduction and preparatory adjustments. Earthquake Commission, New Zealand

Paton D, Smith L, Johnston DM (2005) When good intentions turn bad: promoting natural hazard preparedness. Aust J Emerg Manag 20(1):25-30

Paton D, Bajek R, Okada N, McIvor D (2010) Predicting community earthquake preparedness: a crosscultural comparison of Japan and New Zealand. Nat Hazards 54:765-781

Perez-Fuentes G, Joffe H (2015) An intervention to increase earthquake and fire preparedness. Paper presented at the sustainable development conference proceedings

Riggs ML, Knight PA (1994) The impact of perceived group success-failure on motivational beliefs and attitudes: a causal model. J Appl Psychol 79:755

Russell LA, Goltz JD, Bourque LB (1995) Preparedness and Hazard Mitigation Actions before and after 2 earthquakes. Environ Behav 27:744-770. doi:10.1177/0013916595276002

Rüstemli A, Karanci AN (1999) Correlates of earthquake cognitions and preparedness behavior in a victimized population. J Soc Psychol 139:91-101

Scawthorn C, O'Rourke T, Blackburn F (2006a) The 1906 San Francisco earthquake and fire-enduring lessons for fire protection and water supply. Earthq Spectra 22:135-158

Scawthorn C, Schneider PJ, Schauer BA (2006b) Natural hazards-the multihazard approach. Nat Hazards Rev 7:39-39

Simpson DM (2002) Earthquake drills and simulations in community-based training and preparedness programmes. Disasters 26:55-69

Sjoberg L (1999) Consequences of perceived risk: demand for mitigation. J Risk Res 2:129-149

Solberg C, Rossetto T, Joffe H (2010) The social psychology of seismic hazard adjustment: re-evaluating the international literature. Nat Hazards Earth Syst Sci 10:1663-1677

Speer PW, Peterson NA (2000) Psychometric properties of an empowerment scale: testing cognitive, emotional, and behavioral domains. Soc Work Res 24:109-118

Spittal MJ, Walkey FH, McClure J, Siegert RJ, Ballantyne KE (2006) The Earthquake Readiness Scale: the development of a valid and reliable unifactorial measure. Nat Hazards 39:15-29. doi:10.1007/s11069005-2369-9

Tanes Z, Cho H (2013) Goal setting outcomes: examining the role of goal interaction in influencing the experience and learning outcomes of video game play for earthquake preparedness. Comput Hum Behav 29:858-869 
Tierney KJ, Lindell MK, Perry RW (2001) Facing the unexpected: disaster preparedness and response in the United States. Joseph Henry Press, Washington

Verrucci E et al (2016) Digital engagement methods for earthquake and fire preparedness—a review. Nat Hazards 83:1583

Weinstein ND, Sandman PM (1992) A model of the precaution adoption process: evidence from home radon testing. Health Psychol 11:170 